INFLAMMATORY BOWEL DISEASE

\title{
Synbiotic therapy (Bifidobacterium longum/Synergy 1) initiates resolution of inflammation in patients with active ulcerative colitis: a randomised controlled pilot trial
}

\author{
E Furrie, S Macfarlane, A Kennedy, J H Cummings, S V Walsh, D A O’Neil, G T Macfarlane
}

Gut 2005;54:242-249. doi: 10.1136/gut.2004.044834

See end of article for authors' affiliations

Correspondence to: Dr E Furrie, Microbiology and Gut Biology Group, University of Dundee,

Ninewells Hospital Medical School, Dundee DDI 9SY, UK; e.furrie@dundee.ac.uk

Revised version received 13 May 2004

Accepted for publication

22 June 2004
Background and aims: Ulcerative colitis (UC) is an acute and chronic inflammatory disease of the large bowel with unknown aetiology. The immune response against normal commensal microorganisms is believed to drive inflammatory processes associated with UC. Therefore, modulation of bacterial communities on the gut mucosa, through the use of probiotics and prebiotics, may be used to modify the disease state.

Methods: A synbiotic was developed for use in UC patients combining a probiotic, Bifidobacterium longum, isolated from healthy rectal epithelium, and a prebiotic (Synergy 1), a preferential inulinoligofructose growth substrate for the probiotic strain. Treatment was employed in a double blinded randomised controlled trial using 18 patients with active UC for a period of one month. Clinical status was scored and rectal biopsies were collected before and after treatment, and transcription levels of epithelium related immune markers were measured.

Results: Sigmoidoscopy scores (scale 0-6) were reduced in the test group (start 4.5 (1.4), end 3.1 (2.5)) compared with placebo (start $2.6(2.1)$, end $3.2(2.2))(p=0.06)$. mRNA levels for human beta defensins 2,3 , and 4 , which are strongly upregulated in active UC, were significantly reduced in the test group after treatment $(p=0.016,0.038$, and 0.008 , respectively). Tumour necrosis factor $\alpha$ and interleukin $1 \alpha$, which are inflammatory cytokines that drive inflammation and induce defensin expression, were also significantly reduced after treatment $(p=0.018$ and 0.023 , respectively). Biopsies in the test group had reduced inflammation and regeneration of epithelial tissue.

Conclusions: Short term synbiotic treatment of active UC resulted in improvement of the full clinical appearance of chronic inflammation in patients receiving this therapy.
$\mathrm{U}$ lcerative colitis (UC) is a relapsing inflammatory disease of the colon with unknown aetiology. Evidence from animal models suggests that an altered immune response towards the commensal gut microbiota plays a key role in the development and maintenance of this condition. ${ }^{1-5}$ For example, knockout or transgenic mice with genetic susceptibilities to IBD only acquire characteristic lesions when their colon is populated with normal commensal bacteria while germ free animals do not manifest an inflammatory response. ${ }^{67}$ Evidence from human studies has also suggested that mucosal bacterial populations in UC may be altered towards a more proinflammatory phenotype. ${ }^{2-11}$

Manipulation of the mucosal microbiota to reduce the inflammatory potential of colonising bacteria is therefore an attractive therapy for UC. One option is to use antibiotics to remove species involved in inducing the inflammatory response. However, antibiotic therapy has had limited success in UC, possibly due to the fact that treatment needs to be customised for individual patients. ${ }^{12-16}$ An alternative is to use probiotic bacteria that interact with the host epithelium to resolve inflammation. Probiotics have been defined as live microbial feed supplements that beneficially affect the host by improving its intestinal microbial balance. ${ }^{17}$ The most widely used probiotics in humans are bifidobacteria and lactobacilli but other organisms such as Escherichia coli and the yeast Saccharomyces boulardii have been reported to have some beneficial effects in maintaining remission in inflammatory bowel disease (IBD). ${ }^{18-20}$

Probiotic therapy can be potentially improved through combination with a prebiotic (usually a non-digestible oligosaccharide that is not absorbed in the upper gut) that promotes growth of the probiotic in the large bowel. This combination is known as a synbiotic. A further advantage of using a synbiotic is that the prebiotic component would promote the growth of indigenous organisms in the gut with probiotic properties. ${ }^{21}$ Few clinical trials have been performed with probiotics to treat or maintain remission in IBD and the resulting success has been variable. ${ }^{22-25}$ The best known product, VSL\#3, uses a mixture of eight different bacterial strains that have been reported to prevent the onset of pouchitis after pouch formation in UC patients. ${ }^{26}{ }^{27}$ Assessments of inflammation in UC have traditionally been done by the gastroenterologist on examination of the bowel at endoscopy and by a pathologist who examines microscopic inflammatory responses in biopsies. However, new sensitive methods of measuring changes in gene expression in inflamed tissue have been developed. Proinflammatory cytokines have been measured before in probiotic therapy in ex vivo colonic biopsy specimens from Crohn's patients where decreased expression of tumour necrosis factor $\alpha$ (TNF- $\alpha$ ) occurred, ${ }^{28}$ but these cytokines can be produced by a variety of infiltrating cells and give little indication of the immune responsiveness of the epithelium. ${ }^{29}{ }^{30}$ A newly described group of antimicrobial peptides known as human beta defensins (hBD) are expressed uniquely by epithelial cells. Six hBD (hBDl-6) are currently recognised. hBDl is constitutively expressed and its promoter has no functioning

Abbreviations: $\mathrm{BHI}$, bowel habit index; $\mathrm{CAl}$, clinical activity index; $\mathrm{CRP}$, $\mathrm{C}$ reactive protein; GAPDH, glyceraldehyde-3-phosphate dehydrogenase; HS, histology score; hBD, human beta defensin; IBD, inflammatory bowel disease; IL- $1 \alpha$, interleukin $1 \alpha$; IL-10, interleukin 10; PCR, polymerase chain reaction; SS, sigmoidoscopy score; TNF- $\alpha$, tumour necrosis factor $\alpha$; UC, ulcerative colitis; WC, Wilkins-Chalgren 
response elements; therefore, the level of expression of hBDl mRNA directly allows absolute determination of the number of epithelial cells in each biopsy. ${ }^{31-33}$ hBD2 and 3 have been shown to be significantly upregulated in UC but not in Crohn's disease ${ }^{34-36}$ and are consequently excellent target genes for assessing inflammatory responses in UC epithelia.

In this pilot study, we have developed a synbiotic for treating UC patients and, to our knowledge, this is the first report of synbiotic therapy in the treatment of UC. To investigate the effectiveness of the synbiotic, we used traditional methods of assessing inflammation, in conjunction with new techniques that directly measure the inflammatory state of the gut epithelium in a quantitative way.

The principal aim of this work was to induce clinical improvement in UC patients receiving synbiotic therapy in comparison with the placebo group. The second objective was to assess the effects of synbiotic feeding on the mucosa, with reference to inflammatory mediators induced in active colitis.

\section{METHODS}

\section{Patients}

Consecutive patients with active UC attending the Gastroenterology Outpatients Clinic, Ninewells Hospital, were asked to give written consent to take part in this investigation. Eighteen patients accepted the invitation. Eligible patients were aged 24-67 years who had not received antibiotics in the last three months and were not taking commercially available probiotic preparations. Normal healthy control biopsies were obtained from other patients attending the clinic who had been shown by sigmoidoscopy and histology to have no evidence of inflammatory bowel disease. These studies were approved by the Tayside Committee on Medical Research Ethics, Dundee (study number 205/01).

\section{Study design}

Eighteen study numbers were assigned and randomised using a table of random digits. ${ }^{37}$ Nine patient numbers were assigned to the test group and nine to the placebo group. The 18 patients were randomly assigned to either group, and given a study number ( $\mathrm{SOl}$ to 18 ). This assignment was not divulged to the clinician, patient, or inhouse researcher who carried out the experimental measurements. A description of patient involvement is given in table 1. Two patients did not attend the first study visit because they had taken antibiotics after recruitment, and therefore 16 patients entered the study. Their characteristics are given in table 2. Patients were recruited on the basis of active inflammation; time lag between recruitment and initiation of the study was up to two months. In one case (table 2) a placebo patient had entered remission after recruitment but before commencement of the study and had a sigmoidoscopy score of 0 and a clinical activity index of 1 . Recruits were maintained on the therapy they were receiving at initiation of the trial which was not altered during the study. Each patient was assessed in the IBD research clinic using the clinical activity index ${ }^{38}$ and sigmoidoscopic appearance scored, ${ }^{39}$ as described in table 3 . They were also requested to keep a daily bowel habit

\begin{tabular}{|c|c|c|}
\hline & $\begin{array}{l}\text { Synbiotic } \\
\text { (8 starting } \\
\text { patients) }\end{array}$ & $\begin{array}{l}\text { Placebo } \\
\text { (8 starting } \\
\text { patients) }\end{array}$ \\
\hline Age $(y)$ & $45(24-66)$ & $38(26-59)$ \\
\hline $\operatorname{Sex}(M: F)$ & $3: 5$ & $5: 3$ \\
\hline Duration of disease (y) & $10(1-23)$ & $7(1-15)$ \\
\hline \multicolumn{3}{|l|}{ Disease extent* ${ }^{*}$} \\
\hline Total & 3 & 2 \\
\hline Left sided & 4 & 3 \\
\hline Proctosigmoid & 1 & 2 \\
\hline \multicolumn{3}{|l|}{ Current drugst } \\
\hline Steroids & 5 & 5 \\
\hline Immunosupressants & 6 & 6 \\
\hline 5-ASA & 5 & 5 \\
\hline Initial clinical activity index & $5.6(2-13)$ & $4.7(1-8)$ \\
\hline Initial sigmoidoscopy score & $4.4(2.5-6.0)$ & $2.9(0-6)$ \\
\hline Initial CRP (mg/l) & $6.0(0-16)$ & $5.5(0-17)$ \\
\hline \multicolumn{3}{|c|}{$\begin{array}{l}\text { Values are mean (range) or number. } \\
\text { *Disease extent unknown for one placebo patient. } \\
\text { tUlcerative colitis related drugs only. } \\
\text { CRP, C reactive protein. }\end{array}$} \\
\hline
\end{tabular}

diary, previously validated by Cummings and colleagues, ${ }^{40}$ in which details of the amount and consistency of each stool were recorded, together with the presence of blood or mucus, and whether the subject experienced abdominal pain. Rectal biopsies were also taken for histology scoring by the Department of Pathology, Ninewells Hospital (no inflammation $=0$, mild inflammation $=1$, moderate inflammation $=2$, and severe inflammation $=3$ ). Venous bloods were taken for measurement of $\mathrm{C}$ reactive protein (CRP; Biochemical Medicine, Ninewells Hospital, UK). Further biopsies were used for inhouse assessment of mucosal inflammatory mediators.

Test patients were given $2 \times 10^{11}$ freeze dried viable Bifidobacterium longum in a gelatin capsule and a sachet containing $6 \mathrm{~g}$ of prebiotic fructo-oligosaccharide/inulin mix (Synergy 1; Orafti, Tienen, Belgium), twice daily for four weeks. Placebos were given in an identical capsule, containing potato starch, and sachets of $6 \mathrm{~g}$ of powdered maltodextrose (Orafti) to simulate the prebiotic. The synbiotic/placebo was taken after breakfast and following the evening meal to minimise the inhibitory effects of gastric acid on the probiotic. At the end of one month, each patient was reassessed in the clinic and scored in the same way as the pretreatment visit. Biopsies were taken for histology and measurements of inflammatory markers, while CRP levels were measured as before.

\section{Production of the probiotic}

Nineteen different bifidobacterial isolates were assessed for suitability as a probiotic strain ( 10 were isolated from healthy colonic mucosae, five were obtained from the faeces of healthy donors, and four were culture collection strains). They comprised six strains of $B$ adolescentis, two $B$ bifidum, two $B$ infantis (DSM 86184, ATCC 15617), two B longum, and one

Table 1 Description of patient involvement during the feeding study

\begin{tabular}{lll}
\hline & Synbiotic (9 starting patients) & Placebo (9 starting patients) \\
\hline $\begin{array}{l}\text { Pretreatment } \\
\text { During study }\end{array}$ & $\begin{array}{l}\text { One removed (antibiotics) } \\
\text { No adverse responses }\end{array}$ & $\begin{array}{l}\text { One removed (antibiotics) } \\
\text { Two withdrew due to deterioration of condition } \\
\text { (week 2) }\end{array}$ \\
$\begin{array}{ll}\text { Post-treatment } \\
\text { Remaining 8 patients completed } \\
\text { the study, 7 with full biopsy retrieval }\end{array}$ & $\begin{array}{l}\text { Remaining 6 patients completed study, 5 with full } \\
\text { biopsy retrieval }\end{array}$ \\
\hline
\end{tabular}


Table 3 System used to score inflammation in the colon at sigmoidoscopy (modified from Baron and colleagues ${ }^{39}$ )

\begin{tabular}{llll}
\hline & $\mathbf{0}$ & $\mathbf{1}$ & $\mathbf{2}$ \\
\hline Friability & None & On touching mucosa & - \\
Bleeding & No blood & Blood in lumen & - \\
Vessel pattern & Normal & Some patchy changes & All vessels obscured \\
Overall appearance & Normal & Moderate change & Severe active disease \\
\hline
\end{tabular}

each of $B$ angulatum, $B$ breve, $B$ catenulatum, $B$ dentium, $B$ lactis (DSM 10140), B pseudocatenulatum, and B suis (ATCC 17533). The organisms were assessed for aerotolerance, acid tolerance, bile salt resistance, adhesion to epithelial cells, and their abilities to utilise oligofructose as an energy source. Their abilities to survive freeze drying and long term storage were also determined. The organism which performed best in all cases was a $B$ longum strain isolated from the healthy rectal mucosa. Its identity was confirmed by partial sequencing ( 500 of $1500 \mathrm{bp}$ ) of the variable region of the 16S rRNA gene. Further analysis of the ability of the selected organism to alter the profile of cytokine expresssion in a model epithelial cell line (HT29) was investigated. Proinflammatory cytokine interleukin $1 \alpha$ (IL- $1 \alpha)$ was measured by both real time polymerase chain reaction (PCR) for mRNA levels and cytokine ELISA for secreted proteins, either alone or in coculture with the selected B longum, B bifidum, and a commensal Escherichia coli. The selected probiotic was found to significantly reduce IL- $1 \alpha$ produced by HT- 29 cells with respect to mRNA levels $(p<0.05)$ and protein $(p<0.05)$, unlike the other two organisms which increased expression of this proinflammatory cytokine. The organism was subsequently grown overnight in batch culture under anaerobic conditions at $37^{\circ} \mathrm{C}$ in Wilkins-Chalgren (WC) broth. Bacteria were harvested by centrifugation (14000 g, 30 minutes) and washed twice in anaerobic phosphate buffered saline. The pellet was frozen overnight at $-80^{\circ} \mathrm{C}$ and then lyophilised (Modulyo Bench Top Freeze Dryer; Thermo Savant, Holbrook, New York, USA). The resulting powder was weighed, and in order to determine cell viability and check purity, serial dilutions were made and plated onto WC agar plates. These were incubated aerobically and anaerobically to maximise contaminant detection. All quantitation and purity tests were checked by two independent microbiologists during production and packaging of the probiotic.

\section{RNA and CDNA preparation}

Biopsies were macerated using liquid nitrogen snap freezing and mechanical grinding. RNA was purified using the RNA easy kit (Qiagen, Hilden, Germany) with an initial clean-up stage using a Qiashredder column (Qiagen) and an additional step of DNA digestion to ensure no genomic DNA contamination. Samples were reverse transcribed using the AMV RT kit (Promega, Madison, Wisconsin, USA) according to the manufacturers instructions and aliquoted before storage at $-80^{\circ} \mathrm{C}$.

\section{Preparation of standards for quantitation of DNA}

Standard amounts of DNA corresponding to the target sequences are needed to carry out real time PCR. This was achieved by making purified plasmid DNA containing the target sequences. Briefly, cDNA from normal healthy colon, or mononuclear blood cells, was amplified using the specific PCR primer pair (see table 4). Product of correct size and sequence was purified using the Qiaquick PCR purification kit (Qiagen) and ligated into a vector using the pGEM-T easy vector system I (Promega). JM109 competent E coli (Promega) were transformed with each ligated vector, and after overnight incubation positive colonies were chosen. From each selected colony the plasmid was purified using the Wizard plus SV miniprep system (Promega). Concentration of the plasmid preparation was determined by agarose gel electrophoresis with known standards (New England Biolabs, Beverly, Massachusetts, USA). Samples were diluted to $10^{10}$ molecules $/ \mu \mathrm{l}$, aliquoted, and stored $\left(-80^{\circ} \mathrm{C}\right)$.

\section{Real time quantitative PCR}

The appropriate plasmid preparation was diluted to give a standard curve of $10^{6}-10^{1}$ molecules/ $\mu$ l for all assays, except glyceraldehyde-3-phosphate dehydrogenase (GAPDH) which had a standard curve of $10^{8}-10^{1}$ molecules/ $\mu$ l. Real time PCR

\begin{tabular}{|c|c|}
\hline Gene target & Primer pair $5^{\prime}$ to $3^{\prime}$ \\
\hline Glyceraldehyde-3-phosphate dehydrogenase (GAPDH) & $\begin{array}{l}\text { GGAAGGTGAAGGTCGGAGTC } \\
\text { CTCAGCCTTGACGGTGCCATG }\end{array}$ \\
\hline Human beta defensin 1 (hBDI) & $\begin{array}{l}\text { CTCTGTCAGCTCAGCCTC } \\
\text { CTTGCAGCACTTGGCCTTCCC }\end{array}$ \\
\hline Human beta defensin 2 (hBD2) & $\begin{array}{l}\text { CCAGCCATCAGCCATGAGGGT } \\
\text { GGAGCCCTTTCTGAATCCGCA }\end{array}$ \\
\hline Human beta defensin 3 (hBD3) & $\begin{array}{l}\text { CGGCCACGCGTCGAGCACTTG } \\
\text { CTCCTITGGAAGGCAGCTGAG }\end{array}$ \\
\hline Human beta defensin 4 (hBD4) & $\begin{array}{l}\text { GTGGAGCCATATGTCATC } \\
\text { CTGAATCCGCATCAGCC }\end{array}$ \\
\hline Interleukin $1 \alpha(\mathrm{IL}-1 \alpha)$ & $\begin{array}{l}\text { ATGGCCAAAGTTCCAGACATGTाT } \\
\text { TIGGTCTCATCTTGGGCAGTCAC }\end{array}$ \\
\hline Tumour necrosis factor $1 \alpha(\mathrm{TNF}-\alpha)$ & $\begin{array}{l}\text { TCTCGAACCCCGAGTGACAA } \\
\text { TATCTCTCAGCTCCACGCCA }\end{array}$ \\
\hline Interleukin 10 (IL-10) & $\begin{array}{l}\text { TCAGGGTGGCGACTCTAT } \\
\text { GTCCGGGGTGCATATCTCTACAGT }\end{array}$ \\
\hline Bifidobacterial genus specific & $\begin{array}{l}\text { AGGGTTCGATTCTGGCTCAG } \\
\text { CATCCGGCATTACCACCC }\end{array}$ \\
\hline
\end{tabular}




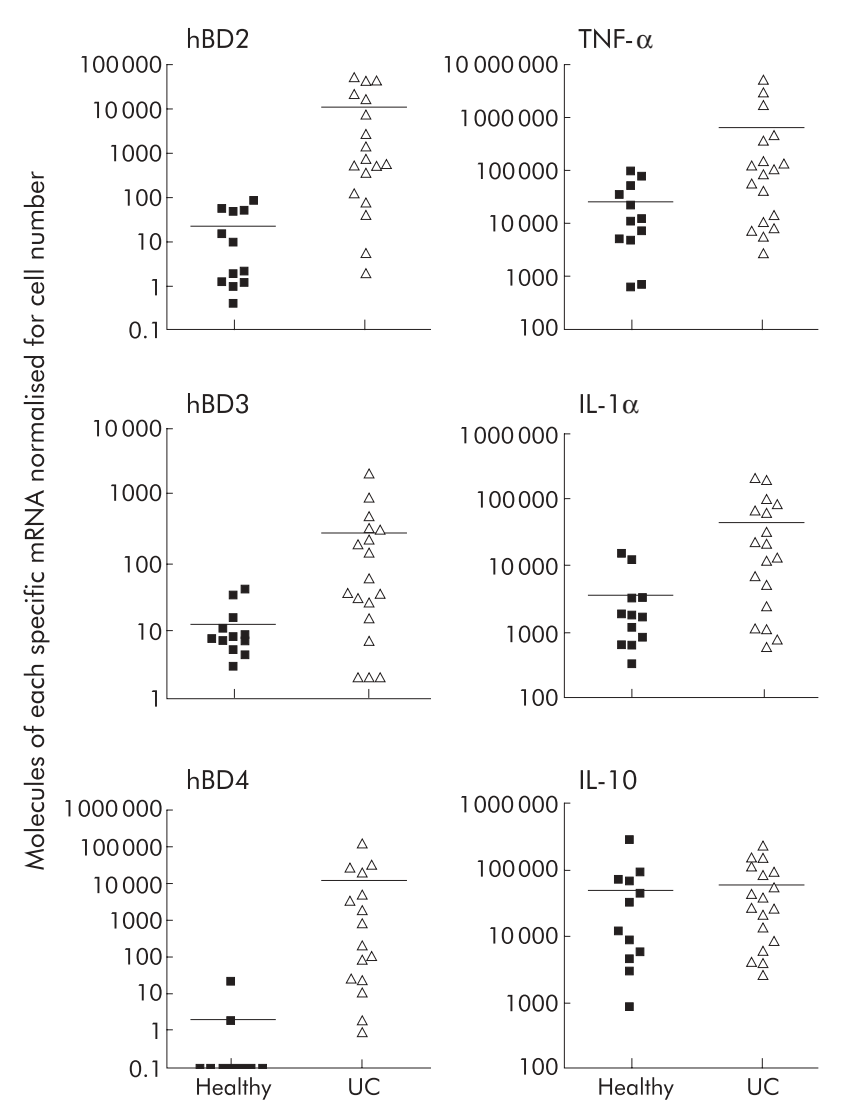

Figure 1 Comparison of human beta defensin (hBD)2 $(p<0.0001)$, hBD3 $(p=0.0010), h B D 4(p<0.0001)$, tumour necrosis factor $\alpha$ (TNF- $\alpha)$ $(p=0.0150)$, interleukin $1 \alpha(\mathrm{IL}-1 \alpha)(p=0.0089)$ ?, and interleukin 10 (IL10) mRNA in ulcerative colitis (UC) rectal biopsies $(n=18)$ versus normal rectal biopsies $(n=12)$. Bars represent mean (SD) and results were normalised for epithelial cell numbers through expression levels of $h B D 1$ for inducible hBD (2-4), and for total cells through glyceraldehyde-3phosphate dehydrogenase levels for cytokines.

was carried out using an iCycler and the iQ SYBR Green Supermix (BioRad, Hercules, California, USA). Test samples were added in triplicate at $2 \mu \mathrm{l} /$ well in a $20 \mu \mathrm{l}$ total reaction volume.

\section{Histopathology}

Tissue specimens were fixed in formalin and embedded in paraffin. Sections ( $4 \mu \mathrm{m}$ thick) were cut using a microtome (Leica RM 2135) and mounted onto clean glass slides which had been coated with poly-L-lysine (Superfrost plus; VWR International Ltd, Poole, Dorset, UK) to improve tissue adherence. Four serial sections were stained with haematoxylin and eosin, using standard methods, to visualise tissue morphologies.

\section{Statistics}

Significant differences between the pre-synbiotic and postsynbiotic therapy groups and between the post-synbiotic and post-placebo groups were assessed for hBD and cytokine results using the Mann-Whitney test for non-parametric analysis as the data were not normally distributed. Significant difference in clinical outcome was assessed using the difference in sigmoidoscopy score (SS) in the synbiotic group over four weeks compared with the difference in SS in the placebo group using a two sample $t$ test with equal variances. Significance was given for $\mathrm{p}$ values less than 0.05 .

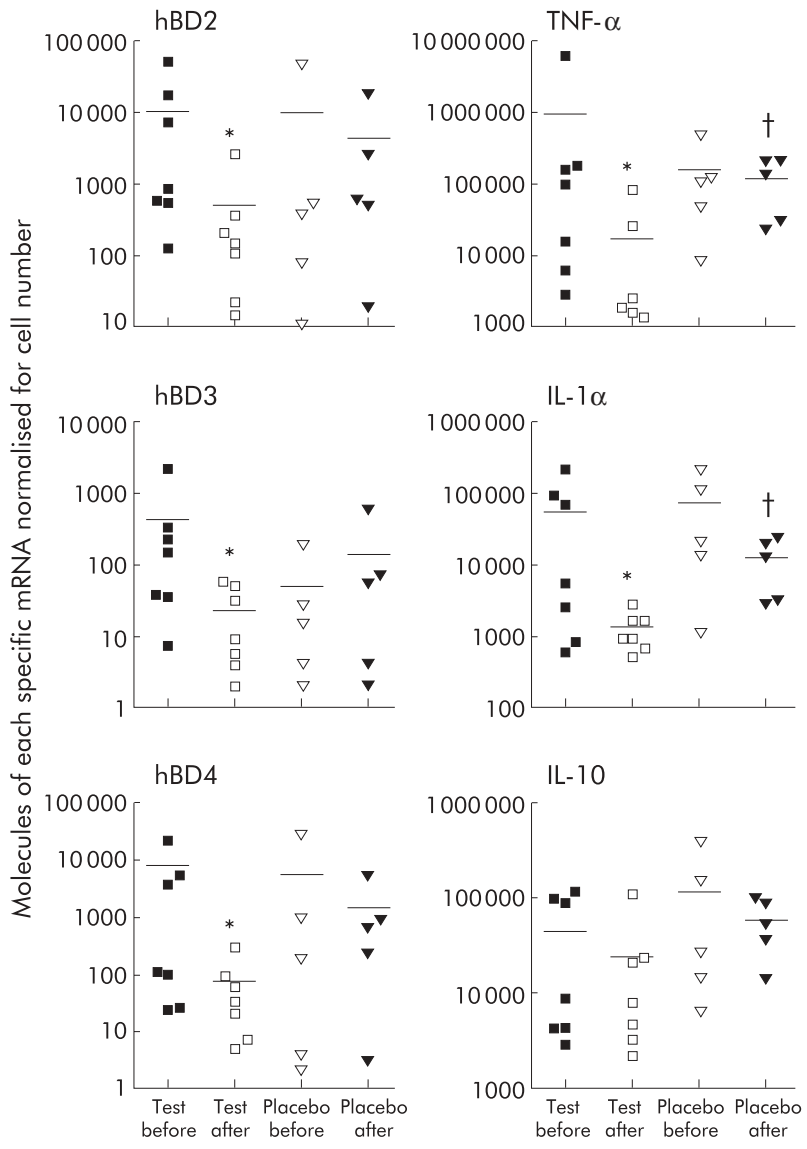

Figure 2 Human beta defensin (hBD) 2-4 and cytokine (tumour necrosis factor $\alpha$ (TNF- $\alpha)$, interleukin $1 \alpha($ IL-1 $\alpha)$, interleukin 10 (IL-10)) mRNA concentrations in mucosal tissue before and after four weeks' consumption of synbiotic or placebo. Bars represent means (SD). All results shown for inducible $h B D(2-4)$ were normalised for epithelial cell numbers, as determined by levels of hBDI, the constitutive epithelial cell housekeeping gene, and cytokine levels were normalised for total cells per biopsy using glyceraldehyde-3-phosphate dehydrogenase. Significance between the pre- and post-synbiotic groups were hBD2 $\left({ }^{*} p=0.0156\right), h B D 3\left({ }^{*} p=0.0379\right), h B D 4\left({ }^{*} p=0.0078\right)$, TNF- $\alpha$ $\left({ }^{*} p=0.0175\right), I L-1 \alpha\left({ }^{*} p=0.0379\right)$, and IL-10 (NS). Significance between the post-synbiotic and post-placebo groups were hBD2 (NS), hBD3 (NS), hBD4 (NS), TNF- $\alpha(t p=0.0177), I L-1 \alpha(t p=0.0051)$, and IL-10 (NS).

\section{RESULTS}

Inflammatory markers in healthy and UC tissues

All results shown for inducible hBD (2-4) were normalised for epithelial cell numbers, as determined by levels of hBDl, the constitutive epithelial cell housekeeping gene. Figure 1 shows that there were significant increases in mRNA expression for all inducible defensins measured (hBD2 $\mathrm{p}<0.0001, \mathrm{hBD} 3 \mathrm{p}=0.0010, \mathrm{hBD} 4 \mathrm{p}<0.0001)$ in UC rectal biopsies $(n=18)$ compared with normal healthy rectal biopsies $(n=12)$. Analysis of the proinflammatory cytokines TNF- $\alpha$ and IL- $1 \alpha$ were also significantly increased in UC compared with normal controls $(p=0.0150$ and $p=0.0089$, respectively) while there was no significant difference in the immunomodulatory cytokine interleukin 10 (IL-10).

\section{Effect of synbiotic on inflammatory markers in the gut} Figure 2 shows the results from the two trial groups, before and after the four week feeding period. Significant differences in expression of inducible hBD occurred in the synbiotic patients ( $\mathrm{hBD} 2 \mathrm{p}=0.0156, \mathrm{hBD} 3 \mathrm{p}=0.0379$, hBD4 $\mathrm{p}=0.0078$ ). No significant differences between the postsynbiotic group and the post-placebo were observed for hBD 

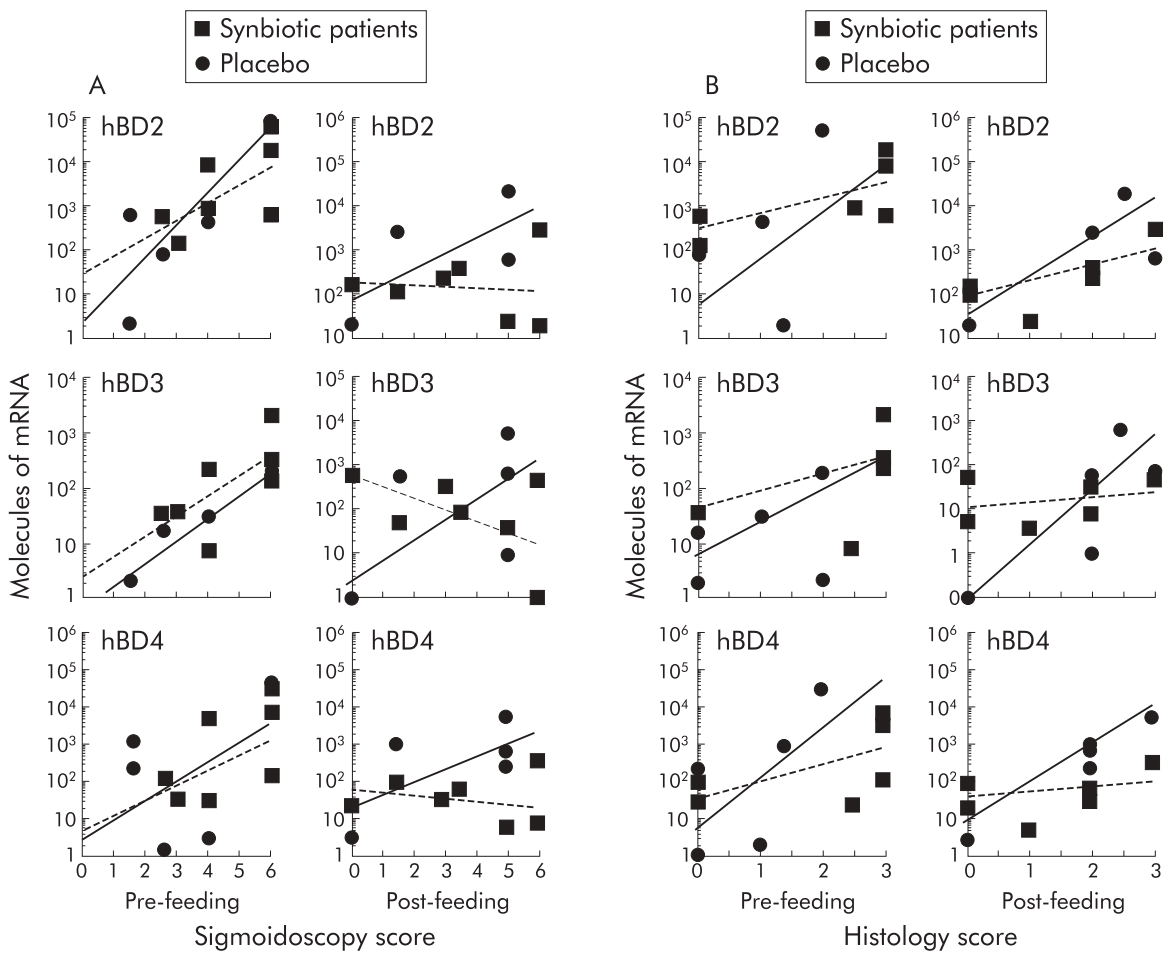

Figure 3 Comparison of sigmoidoscopy scores (scale $0-6$ ) with inducible human beta defensin (hBD) mRNA synthesis (A) and histology scores (scale $0-3$ ) with inducible hBD mRNA synthesis (B) in synbiotic patients and placebos. Lines represent exponential best fits for synbiotic (broken lines) and placebo (solid lines) data sets, respectively.

due to the small number of placebos who completed the trial. Similar results were obtained for the proinflammatory cytokines TNF- $\alpha$ and IL- $1 \alpha$, with significant reductions in expression in the post-feeding synbiotic patients compared with the pre-synbiotic group $(p=0.0175$ and $p=0.0379$, respectively). On comparison of the levels of inflammatory cytokines for the post-synbiotic group versus the post-placebo group, we found a significant reduction for both TNF- $\alpha$ and IL- $1 \alpha$ levels $(p=0.0177$ and $p=0.0051$, respectively). The most marked reduction was seen with IL- $l \alpha$, which returned to levels found in normal healthy tissue (see fig 1). No significant difference was seen in IL-10 between the placebo and synbiotic groups.

\section{Presence of bifidobacteria on the mucosa}

Levels of bifidobacterial specific total rRNA were determined using real time PCR in mucosal biopsies pre- and posttreatment. The synbiotic group had a starting mean of 1406 molecules of bifidobacterial rRNA per biopsy, which rose to a mean of 58699 at the end of the study, a 42 -fold increase. The placebo group had a mean starting level of 3527 bifidobacterial rRNA copies per biopsy increasing to 16285 over the same four week interval, a 4.6-fold increase. Samples were normalised for number of epithelial cells by hBDl levels.

\section{Clinical outcome}

Clinical outcome for patients was assessed by sigmoidoscopy score (SS), clinical activity index (CAI), and bowel habit index (BHI). Results are expressed as the difference in values recorded on day 1 and day 28 of the trial for SS and CAI, and as the percentage change over the four week period for BHI. SS (maximum change possible $=6$ ) was decreased by a mean of 1.3 points over the four week period in the synbiotic group $(\mathrm{n}=8)$; five patients improved, two remained the same, and one increased from 4 to 5 . The placebo group had an increase of 0.58 over the same period $(n=6)$; three had increased scores and three had decreased scores. SS was reduced markedly in the synbiotic group after treatment (start 4.5 (1.4), end $3.1(2.5))$ compared with the placebo group $(n=6$; start $2.6(2.1)$, end $3.2(2.2)$ ). The difference in pre- and postfeeding between the synbiotic and placebo groups was borderline significant $(p=0.06)$. CAI (maximum score 19) was reduced in five patients in the synbiotic group with three showing an increase in score (start 5.6 (3.7), end 5.3 (3.4)). The placebo group had three patients with improving scores, one which remained the same and two with increased CAI (start 4.9 (3.2), finish $3.1(2.5)$ ). Mean BHI increased in the placebo group by $70.4 \%$ compared with a reduction of $20.4 \%$ in the synbiotic group.

\section{Correlation of hBD levels with sigmoidoscopy scores (SS)}

Analysis of the relationship between SS and levels of individual hBD showed that there was no correlation between hBDl with SS, before or after treatment, in either the test or placebo group (results not shown). However, in both synbiotic and placebo groups pretreatment, there was a positive correlation between SS and mRNA for hBD2-4 whereby in more active disease higher levels of inducible hBD were detected (fig 3A). At the end of the feeding study, comparison of SS and hBD showed that this positive correlation remained only in the placebo group whereas hBD2 and hBD4 manifested a loss of correlation with SS score, and hBD3 had an inverse relationship in the synbiotic group.

\section{Correlation of hBD levels with histology scores (HS)} A positive correlation was evident in the relationship between HS and hBD2-4 in both synbiotic and placebo groups, before 


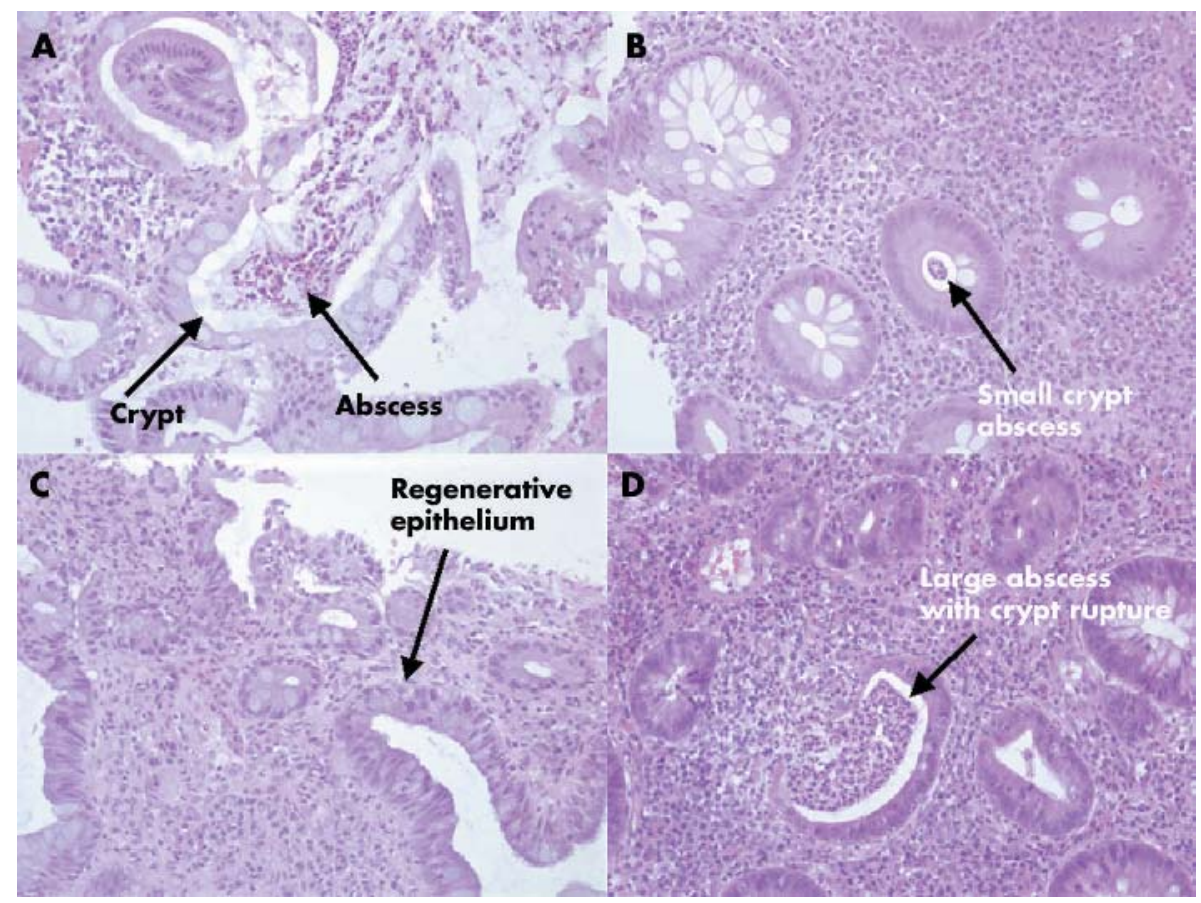

Figure 4 Representative histopathology of rectal mucosa from a patient with ulcerative colitis pre-synbiotic therapy (A) and post-treatment (C), compared with a placebo patient at the start (B) and the end (D) of the study.

the feeding study (fig 3B). After treatment, the placebos retained a positive correlation for all three inducible beta defensins, as occurred with SS. In the synbiotic group, a positive correlation for hBD2 and 4 remained when comparing HS with mRNA concentrations but hBD3 levels related poorly with HS in this group (fig 3B).

\section{$C$ reactive protein (CRP) levels in blood}

Only five of the eight synbiotic patients had elevated CRP (mean 6.0 (SD 6.5)), and only one of six patients in the placebo group who completed the study had elevated CRP (mean 1.6 (SD 3.6)). After treatment, the synbiotic group showed a reduction in CRP (mean 1.8 (SD 3.9)) after four weeks. None of the placebo patients had raised circulating levels of CRP.

\section{Histopathology of rectal biopsies}

Histology scoring produced results similar to SS; four individuals showed a reduction in score and two remained the same, with a mean reduction in HS in the synbiotic group over the four week period (start 1.7 (1.4), end 1.1 (1.2)) compared with the mean increase in the placebo group (start 0.9 (0.9), end $1.9(1.1)$ ), with three showing an increase in score and two remaining the same. Two representative paired biopsies are shown in fig 4: $\mathrm{A}(\mathrm{SS}=6, \mathrm{HS}=3)$ and $\mathrm{C}$ $(\mathrm{SS}=3.5, \mathrm{HS}=1.5)$ were from a synbiotic patient, pre- and post-feeding, respectively, while $\mathrm{B}(\mathrm{SS}=1.5, \mathrm{HS}=1.5)$ and D ( $S S=5.0, H S=2.5$ ) were from a placebo treated patient, at the beginning and end of the study. Comparison of fig $4 \mathrm{~A}$ and $\mathrm{C}$ shows resolution of acute inflammatory activity following synbiotic consumption. The crypt abscesses in fig 4A have disappeared and the epithelium shows a more regenerative appearance in fig 4C. Small crypt abscesses are evident in the rectal biopsy from the placebo patient (fig 4B). This inflammation has been exacerbated in fig $4 \mathrm{D}$, with a larger abscess visible in the middle of the section, with crypt rupture, and a general increase in the numbers of infiltrating cells.

\section{DISCUSSION}

Consumption of synbiotic twice daily over four weeks significantly reduced mucosal inflammatory markers in active UC. This was concurrent with a reduction in colitis at the macroscopic and microscopic level. However, although lowered SS were observed in the synbiotic group, they were not as marked as the reductions in HS and inflammatory markers. This may indicate that changes in inflammatory mediators at the molecular level could precede gross clinical changes scored through sigmoidoscopy by several weeks. This contention is supported by the relationship seen in HS and not SS after synbiotic feeding, with both hBD2 and 4 showing that although local inflammation was resolving in these patients, more general changes in colonic appearance, such as vessel pattern, take longer to return to normal. Alternatively, this therapy may be resolving the local inflammation through direct contact with the epithelium but is insufficient in all cases of UC (three of eight patients receiving synbiotic therapy had no improvement in SS) in reducing the systemic chronic inflammatory condition. It is possible that these patients may improve their clinical scores if this treatment is given in conjunction with other therapies or through selection of a different probiotic organism. Unfortunately, no significant difference in SS or CAI between the synbiotic and placebo groups was found. This may be due to the small number of placebos who completed the study. Two placebos requested withdrawal because of exacerbation of symptoms, and refused to undergo sigmoidoscopy or provide a final biopsy due to discomfort. There were no withdrawals from the synbiotic group after commencement of the study and no reports of adverse reactions.

Markers of active disease in biopsies from UC patients include proinflammatory cytokines such as TNF- $\alpha$, IL-8, IL$1 \alpha$, and IL- $1 \beta .{ }^{29}$ These molecules are upregulated in active UC but they are not specific for the epithelium as the large numbers of infiltrating leucocytes in the mucosa contribute greatly to their formation. ${ }^{28-30}$ When using a probiotic or synbiotic, it is important to be able to assess directly its effects on the epithelial barrier as well as the underlying 
immune system as the epithelium is the first point of host contact for the organisms. Human beta defensins are good indicators of mucosal immunity because they are uniquely expressed by epithelial cells. ${ }^{31}{ }^{32}$ Two types of expression occur: hBDl is a non-inducible constitutively expressed molecule while hBD2-4 are upregulated by bacterial challenge and proinflammatory cytokines. ${ }^{33} 34$ These molecules are produced by the inflammatory infiltrate and by the epithelium itself ${ }^{30}$ directly affecting expression levels of inducible hBD in epithelial cells. IL-1 has been shown to induce upregulation of hBD2 in gastric and colonic epithelial cell lines. ${ }^{34} 41$

In this study, a direct relation between mRNA synthesis for inducible hBD and severity of UC (SS) has been shown for the first time, together with the relationship between inducible hBD and histology scores in UC patients. We were unable to demonstrate a significant difference between the inducible hBD in the post-synbiotic group versus the postplacebo group but the proinflammatory cytokines TNF- $\alpha$ and IL- $1 \alpha$ were significantly reduced in the synbiotic group compared with the placebo group. This can be explained by the relationship between SS and levels of inducible hBD. As our placebo group had lower clinical scores than the synbiotic group, resulting from the small sample size and random assignment to each group, we have inadvertently biased against finding a significant difference in inducible hBD between the two groups due to the direct linear relationship between SS and hBD. This is not true for the inflammatory cytokines that have a more all or nothing expression pattern and can be produced by any infiltrating cell in the biopsy. Furthermore, they are compared against the usual housekeeping genes (GAPD/ $\beta$-actin) for biopsy size standardisation, unlike hBD which are only produced by colonic epithelial cells and epithelial cell numbers can be accurately standardised by hBDl levels.

The functions of these induced hBDs are unknown in UC disease progression. However, hBD are antimicrobial peptides that are particularly good at destroying Gram negative bacteria $^{33}$ so their production may be an attempt by the epithelium to modify the composition of the mucosal biofilm. The bifidobacterial probiotic strain used in this study was found to be insensitive to hBD mediated killing by recombinant hBDl, 2, and 3 (results not shown), and therefore, as part of a therapy for UC this organism would exhibit better survival characteristics on the inflamed mucosa and interact directly with the epithelial surface and modify the immune response. We detected higher numbers of total bifidobacteria on the mucosal surface in patients fed the synbiotic compared with those taking placebo. We cannot separately identify the probiotic organism from the indigenous bifidobacteria that may have been stimulated by inclusion of a prebiotic in the feeding regimen. Bifidobacterium longum is a very complicated organism with at least five different subgroups contained within the species when $16 \mathrm{~S}$ rDNA sequences are compared (data not shown). With the present technology it is impossible to distinguish the probiotic strain from other Bifidobacterium longum in a small pinch biopsy. It is unknown what direct effects probiotic bacteria have on the inflamed epithelium. They could simply be out competing microorganisms involved in disease aetiology for microniches on the gut wall, thereby removing inflammatory stimuli. However, they may also be directly influencing the expression of inflammatory cytokines by the epithelium through ligation of particular pattern recognition receptors on the luminal cell surface, as supported by studies using HT29 cells in preliminary screening experiments with the probiotic. Alternatively, the probiotic could be directly influencing dendritic cells that are present in high numbers in the lamina propria ${ }^{42}$ with access to the epithelial surface, ${ }^{43}$ by downregulating proinflammatory cytokine pathways and stimulating a more immunomodulatory and tolerant immune response.

In conclusion, this short term pilot study has provided the first evidence that synbiotics have the potential to be developed into acceptable therapies for patients suffering from acute UC. A large scale clinical trial is now needed to investigate the long term effects of synbiotic use in inducing and maintaining remission in patients with active disease.

\section{ACKNOWLEDGEMENTS}

We wish to acknowledge the help of Dr Douglas Steinke as a statistical consultant. The work was funded by the Medical Research Council, UK.

\section{Authors' affiliations}

E Furrie, S Macfarlane, A Kennedy, G T Macfarlane, MRC Microbiology and Gut Biology Group, University of Dundee, Ninewells Hospital Medical School, Dundee, UK

J H Cummings, Division of Pathology and Neuroscience, University of Dundee, Ninewells Hospital Medical School, Dundee, UK

S V Walsh, Department of Pathology, Ninewells Hospital and Medical School, Dundee, UK

D A O'Neil, Immunology Unit, Rowett Research Institute, Aberdeen, UK

Conflict of interest: None declared.

\section{REFERENCES}

1 Macdonald TT, Monteleone G, Pender SLF. Recent developments in the immunology of inflammatory bowel disease. Scand J Immunol 2000;51:2-9.

2 Campieri M, Gionchetti P. Bacteria as the cause of ulcerative colitis. Gut 2001;48:132-5.

3 Schultsz C, Van Den Berg FM, Ten Kate FW, et al. The intestinal mucus layer from patients with inflammatory bowel disease harbors high numbers of bacteria compared with controls. Gastroenterology 1999;117:1089-97.

4 Butcher EC. Leukocyte-endothelial cell recognition: three (or more) steps to specificity and diversity. Cell 1991;67:1033-6.

5 Duchmann R, Kaiser I, Hermann E, et al. Tolerance exists towards resident intestinal flora but is broken in active inflammatory bowel disease (IBD). Clin Exp Immunol 1995; 102:448-55

6 Sadlack B, Merz H, Schorle H. Ulcerative colitis-like disease in mice with a disrupted interleukin-2 gene. Cell 1993;75:253-61.

7 Taurog JD, Richardson JA, Croft JT, et al. The germ free state prevents development of gut and joint inflammatory disease in HLA-B27 transgenic rats. J Exp Med 1994; 180:2359-64.

8 Matsuda H, Fujiyama $Y$, Andoh A, et al. Characterisation of antibody responses against rectal mucosa-associated bacterial flora in patients with ulcerative colitis. J Gastroenterol Hepatol 2000;15:61-8.

9 Hartley MG, Hudson MJ, Swarbrick ET, et al. The rectal mucosa associated microflora in patients with ulcerative colitis. J Med Microbiol 1992;36:96-103.

10 Poxton IR, Brown R, Sawyer A, et al. Mucosa-associated bacterial flora in the human colon. J Med Microbiol 1997:46:85-91.

11 Macfarlane S, Furrie E, Cummings JH, et al. Chemotaxonomic analysis of bacterial populations colonizing the rectal mucosa in patients with ulcerative colitis. Clin Infect Dis 2004;38:1690-9.

12 Dickinson RJ, O'Connor HJ, Pinder I, et al. Double blind controlled trial of oral vancomycin as adjunctive treatment in acute exacerbations of idiopathic colitis. Gut 1985;26:1380-4

13 Mantzaris GJ, Hatzis A, Kontogiannis P, et al. Intravenous tobramycin and metronidazole as an adjunct to corticosteroids in acute, severe ulcerative colitis. Am J Gastroenterol 1994:89:43-6.

14 Turunen UM, Farkkila MA, Hakala K, et al. Long-term treatment of ulcerative colitis with ciprofloxacin: a prospective, double-blind, placebo-controlled study. Gastroenterology 1998;115:1072-8.

15 Present DH. Ciprofloxacin as a treatment for ulcerative colitis-not yet. Gastroenterology 1998;115:1289-91.

16 Cummings JH, Macfarlane GT. Is there a role for microorganisms? In: Jewell DP, Warren BF, Mortensen NJ, eds. Challenges in inflammatory bowel disease. Oxford: Blackwell Science, 2001:47-8.

17 Macfarlane GT, Cummings JH. Probiotics, infection and immunity. Curr Opin Infect Dis 2002;15:501-6.

18 Kruis W, Schutz E, Fric P, et al. Double blind comparison of an oral Escherichia coli preparation and mesalazine in maintaining remission of ulcerative colitis. Aliment Pharmacol Ther 1997;1 1:853-8.

19 Rembacken BJ, Snelling AM, Hawkey PM, et al. Non-pathogenic Escherichia coli versus mesalazine for the treatment of ulcerative colitis: a randomised trial. Lancet 1999;354:635-9.

20 Guslandi M, Mezzi G, Sorghi M, et al. Saccharomyces boulardii in maintenance treatment of Crohn's disease. Dig Dis Sci 2000;45:1462-4.

21 Roberfroid $M$. Prebiotics and synbiotics: concepts and nutritional properties. B J Nutr 1998;80:S197-202. 
22 Venturi A, Gionchetti P, Rizzello F, et al. Impact on the composition of the faecal flora by a new probiotic preparation: preliminary data on maintenance treatment of patients with ulcerative colitis. Aliment Pharmacol Ther 1999; 13:1103-8.

23 Prantera C, Scribano ML, Falasco G, et al. Ineffectiveness of probiotics in preventing recurrence after curative resection for Crohn's disease: a randomised controlled trial with Lactobacillus GG. Gut 2002;51:405-9.

24 Laake KO, Line PD, Aabakkan L, et al. Assessment of mucosal inflammation and circulation in response to probiotics in patients operated with ileal pouch anal anastomosis for ulcerative colitis. Scand J Gastroenterol 2003;38:409-14.

25 Hart AL, Stagg AJ, Kamm MA. Use of probiotics in the treatment of inflammatory bowel disease. J Clin Gastroenterol 2003;36:111-19.

26 Gionchetti P, Rizzello F, Helwig U, et al. Prophylaxis of pouchitis onset with probiotic therapy: a double blind, placebo controlled trial. Gastroenterology 2003; 124:1202-9.

27 Mimura T, Rizzello F, Helwig U, et al. Once daily high dose probiotic therapy (VSL\#3) for maintaining remission in recurrent or refracrory pouchitis. Gut 2004;53:108-14.

28 Borruel N, Carol M, Casallas F, et al. Increased mucosal tumour necrosis factor a production in Crohn's disease can be down regulated ex vivo by probiotic bacteria. Gut 2002;51:659-64.

29 Woywodt A, Ludwig D, Neustock P, et al. Mucosal cytokine expression, cellular markers and adhesion molecules in inflammatory bowel disease. Eur J Gastroenterol Hepatol 1999; 11:267-76.

30 Daig R, Rogler G, Aschenbrenner E, et al. Human intestinal epithelial cells secrete interleukin-1 receptor antagonist and interleukin-8 but not interleukin1 or interleukin-6. Gut 2000:46:350-8

31 Zhao C, Wang I, Lehrer RI. Widespread expression of beta defensin hBD-1 in human secretory glands and epithelial cells. FEBS Lett 1996;396:319-25.
32 Yamaguchi $Y$, Nagase T, Makita R, et al. Identification of multiple novel epididymis-specific beta-defensin isoforms in humans and mice. J Immunol 2002; 169:2516-23

33 O'Neil DA. Regulation of expression of beta-defensins: endogenous enteric peptide antibiotics. Mol Immunol 2003:40:445-50.

34 O'Neil DA, Porter EM, Elewaut D, et al. Expression and regulation of the human beta-defensins hBD-1 and hBD-2 in intestinal epithelium. J Immunol 1999;163:6718-24.

35 Wehkamp J, Harder J, Weichenthal $M$, et al. Inducible and constitutive beta defensins are differentially expressed in Crohn's disease and ulcerative colitis. Inflamm Bowel Dis 2003;9:215-23.

36 Fellerman K, Wehkamp J, Herrlinger KR, et al. Crohn's disease: a defensin deficiency syndrome? Eur J Gastroenterol Hepatol 2003;15:627-34.

37 Daniel WW. Biostatistics: A foundation for analysis in the health sciences, 4th edn. New York: John Wiley and Sons Inc, 1987.

38 Walmsley RS, Ayres RCS, Pounder RE, et al. A simple clinical colitis activity index. Gut 1998;43:29-32.

39 Baron JH, Connell AM, Lennard-Jones JE. Variation between observers in describing mucosal appearances in proctocolitis. BMJ 1964;1:89-92.

40 Cummings JH, Christie S, Cole TJ. A study of fructoloigosaccharides in the prevention of travellers' diarrhoea. Aliment Pharmacol Ther 2001;15:1139-45.

41 O'Neil DA, Cole SP, Martin-Porter E, et al. Regulation of human betadefensins by gastric epithelial cells in response to infection with Helicobacter pylori or stimulation with IL-1. Infect Immun 2000;68:5412-15.

42 Stagg AJ, Hart AL, Knight SC, et al. The dendritic cell: its role in intestinal inflammation and relationship with gut bacteria. Gut 2003;52:1522-9.

43 Rescigno M, Urbano M, Valzasina B, et al. Dendritic cells express tight junction proteins and penetrate gut epithelial monolayers to sample bacteria. Nat Immunol 2001;2:361-7.

\section{EDITOR'S QUIZ: GI SNAPSHOT}

\section{Answer}

From question on page 206

Computed tomography scan of abdomen revealed a large collection of intrahepatic gas peripherally (fig 1) and dilated loops of intestine with pneumatosis (fig 2). Hypotension persisted despite aggressive volume resuscitation. Exploratory laparotomy revealed necrotic intestine from the proximal jejunum to the hepatic flexure. The necrotic intestine was resected, and jejunotransversostomy was performed.

Intrahepatic gas may represent either an incidental finding or a life threatening condition. Intrahepatic gas may be present in either the biliary or portal venous systems. Computed tomography scan may help differentiate gas in the biliary system from that of the portal venous vasculature. Biliary gas tends to be located centrally in the hilar region in the larger bile ducts. In contrast, gas in the portal system extends to the smaller portal venous branches, resulting in a less localised peripheral distribution. Mucosal and submucosal bowel wall damage may allow some intraluminal air to enter the damaged or dissected bowel wall, leading to pneumatosis, and also to enter some intramural veins, leading to portal venous gas. Therefore, portal venous gas may be observed in any patient with severe inflammation of the intestinal walls-for example, necrotising enterocolitis, ulcerative colitis, Crohn's disease, large ulcerating gastric carcinoma, appendicitis, and diverticulitis.

doi: $10.1136 /$ gut.2004.046441

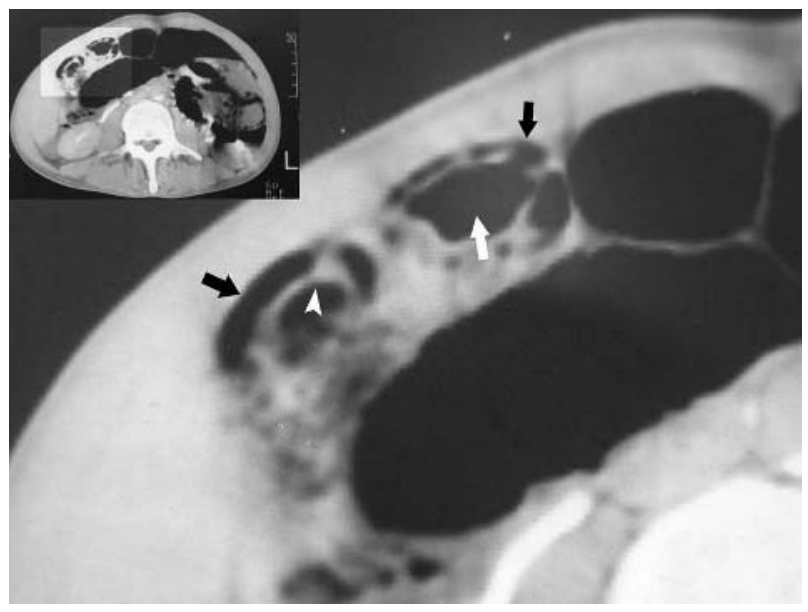

Figure 2 Computed tomography scan demonstrating gas collections in the intestinal wall (black arrows), and a thickened oedematous intestinal wall (arrowhead). White arrow represents luminal air. 